\title{
The Novel Coronavirus (COVID-19) Pandemic and the Response in Low-to-Middle Income Countries
}

\author{
Hania Shahzad ${ }^{1} \cdot$ Fatima Mubarik $^{2}$ - Abida K. Sattar ${ }^{3}$ (D) \\ Accepted: 9 March 2021 / Published online: 16 April 2021 \\ (C) The Author(s), under exclusive licence to Springer Science+Business Media, LLC, part of Springer Nature 2021
}

\begin{abstract}
Purpose of Review The COVID-19 pandemic has posed an unprecedented challenge to healthcare, particularly in resourceconstrained low and middle-income countries (LMICs). We aim to summarize the challenges faced by LMICs in providing breast cancer care during the pandemic and their response during this crisis.

Recent Findings Conversion of oncology centers into COVID-19 isolation centers and lack of LMIC applicable guidelines for breast cancer treatment worsened the challenge for providers. Few LMICs changed their management framework, taking steps like triaging patients, prioritizing care, therapeutic spacing, and a shift to telehealth.

Summary Modified protocols where available have served LMICs well for resource allocation; however, effectiveness of these cannot be determined due to lack of outcomes reporting. This pandemic has underscored the importance of flexibility, prompt intervention, good communication, and reassessment to address unexpected healthcare challenges and has been a learning lesson to help tailor guidelines early in the future.
\end{abstract}

Keywords Corona virus · Pandemic · COVID-19 · Low-to-middle income (LMIC) · Breast cancer · Guidelines

\section{Introduction}

The World Health Organization (WHO) declared the novel coronavirus (COVID-19) a pandemic on March 10, 2020, and as of September 20, 2020, the world has seen more than 30,000,000 confirmed cases and approximately 960,000 deaths attributed to it. This pandemic has thus been a tremendous challenge for the

This article is part of the Topical Collection on Breast Cancer Management during the COVID-19 Pandemic

Abida K. Sattar

abida.sattar@aku.edu

Hania Shahzad

hania.shahzad1995@gmail.com

Fatima Mubarik

fatimamub9@gmail.com

Aga Khan University, Karachi, Pakistan

2 Medical College, Aga Khan University, Karachi, Pakistan

3 Department of Surgery, Aga Khan University, Link Building, Stadium Road, Karachi 74800, Pakistan entire global healthcare community and much worse for low and middle-income countries (LMICs).

The existing, frail healthcare infrastructure of LMICs was already burdened with communicable and non-communicable diseases when they suddenly got challenged with the COVID19 pandemic. Cancer, a significant contributor to the global of non-communicable diseases, accounts for $7.6 \%$ of all global burdern of disability-adjusted life-years [1] with breast cancer as the leading cause of morbidity and mortality. According to GLOBOCAN 2018, breast cancer accounted for one in 4 new cases of cancer in women, contributing $15 \%$ of all female deaths due to cancer [2]. Chinese and Italian studies report that $8.3 \%$ to $29 \%$ of their breast cancer patients contracted COVID-19 [3, 4]. Even though direct causality between cancer and increased risk of contracting COVID-19 infection has not been established, the immunodeficient state of cancer patients receiving chemotherapy may be a predisposing factor. Cancer patients with multiple comorbidities are especially vulnerable to viral infections. Therefore, amidst the pandemic, it is essential to find a fine balance between minimizing exposure of patients to high-risk areas such as healthcare facilities, judiciously using healthcare resources, and providing optimal cancer care. 
LMICs, even on a good day, are resource deficient and certainly not prepared to deal with a healthcare crisis of this magnitude despite a lower disease burden of COVID-19 infections [5]. Though the healthcare community, and specifically leading oncologic societies such as the American Society of Clinical Oncology (ASCO) and the European Society of Medical Oncology (ESMO), put together triaging recommendations to best utilize resources without compromising care, these guidelines are not applicable to all LMICs, as each country struggles with its unique challenges. Countries face difficulties in comprehensive screening and isolation of patients as many patients hide their COVID-19 positivity status due to a fear of social stigma. In regions with political unrest, such as Gaza, patients face additional difficulties in commuting to urban areas for treatment [6]. Limited literature is available that is specifically applicable to resource-limited settings. Hence arises the need to review the continuum of breast cancer care, including challenges faced, responses implemented, and outcomes reported from LMICs.

\section{Global Impact of COVID-19 Infection on Breast Cancer}

As of September 20, 2020, the WHO reported the Americas to be the region most affected by COVID-19, with South-East Asia following closely behind [7]. The actual situation may differ based on the quality and quantity of screening, testing, and outcomes reporting in these regions. Case fatality rates (CFR) continue to change as newer data emerge for each country. A head-to-head comparison of CFRs across different regions may be difficult, affected by inconsistent case definitions and testing strategies. Variation in quality of care or interventions being introduced at different stages of the illness may also play a role. Finally, the profile of patients (for example their age, sex, ethnicity, and underlying comorbidities) may vary between countries [8]. Regardless, the CFR of COVID-19 appears to be lower than that of SARS (9.5\%) but higher than that of influenza $(0.1 \%)$ [9-11]. As the pandemic evolves, there is ongoing research into the relationship between cancer and the COVID-19 infection.

Studies from China have estimated a ten-times higher mortality rate for COVID-19 in cancer patients compared to the general population. Additionally, the use of anticancer therapies such as chemotherapy and radiation within 14 days of infection is reported as an independent predictor of death or other severe events with a hazard ratio $>4$. The same study also reported that a high proportion $(28.6 \%)$ of patients acquire the COVID-19 infection while already in the hospital for cancer treatment [4]. Data from Italy showed that $19.5 \%$ of those who died from COVID-19 had active cancer in the last 5 years [12]. The above-mentioned numbers provide evidence that while a direct link between cancer and the infection is yet to be established, cancer patients may be at increased risk for contracting the disease and for poorer outcomes. Cancer patients infected with the COVID-19 virus have also shown higher rates of hospitalization, admission to intensive care units, and need for invasive tracheal intubations [13] based on Western data. An international COVID-19 and cancer consortium registry analysis of 928 confirmed cases of COVID19 diagnosed within the previous month, who also had a history of active or previous malignancy, found breast cancer to be the most prevalent malignancy (21\%) [14].

Some international organizations provided basic recommendations to mitigate cancer care in resource-limited settings which have been summarized below. To the best of our knowledge, among the WHO listed LMICs, only Pakistan and Morocco shared guidelines that catered specifically to breast cancer patients to provide optimal cancer care in COVID-19 pandemic settings. These LMICs focused on their challenges and tried to optimize the standard protocols for breast cancer care to meet the requirements of these challenging times.

\section{Guidance Available for Management and Outcomes Reported During the COVID-19 Pandemic}

The American Society of Clinical Oncology (ASCO) published recommendations for oncological care in low resource settings in the COVID-19 era. The eleven-point correspondence suggested the need to limit face-to-face contact by adopting virtual assistance using free platforms such as Skype or Google Suite, the need to prioritize care and to consider switching chemotherapy/immunotherapy schedules to space them out in selected cases. Oral therapies were recommended to replace intravenous treatments in advanced cases. Non-critical therapies including the use of bisphosphonates were to be considered for temporary discontinuation and strict selection criteria for in-hospital chemotherapy were suggested. Screening for COVID-19 for all patients visiting the cancer center and protective measures for the healthcare team and a strict protocol for the disinfection of personal devices were recommended. It was also suggested that entry into the hospitals/institutions for cancer care should be monitored and patients should be screened using strict criteria based on their diagnosis per guidelines established by each institution's infectious disease, bioethics, and intensive care unit departments. The correspondence further advised the creation of a population-based registry of COVID-19-positive patients with co-existing cancer to monitor outcomes and refine institutional protocols [15].

A critical review published on advances in radiation oncology explored the mortality risk of presenting to the clinic for treatment and being exposed to infection versus the benefit of 
radiotherapy (RT) itself. It was recommended that physicians consider omitting RT when appropriate, delaying radiation while initiating hormone therapy in low-risk patients with ER positive breast cancer, and adopting accelerated schemes when possible in a concerted effort to protect communities and conserve scarce healthcare resources [16].

Some reviews explored the applicability of cost-effective preventive measures such as using reusable cloth masks in public, saving surgical masks for use within the health facilities, and devising educational materials and programs for increasing awareness in cancer patients regarding proper hygiene and infection prevention measures [17].

The recommendations put forward by various societies for the management of breast cancer during the pandemic were based on expert opinion/best practice. Though in certain cases, delaying chemotherapy was suggested, a 4-week delay in adjuvant chemotherapy for breast cancer has been associated with inferior survival, especially for triple-negative breast cancer (TNBC) [18].

Most of these reports are from the developed world as LMICs were likely overwhelmed and unable to report outcomes expeditiously. In LMICs, breast cancer (BC) patients often present at a younger age, with more aggressive histological subtypes and with advanced-stage disease [19]. Compared to stage I and II early breast cancers, advanced stages demand higher resource allocation for management which include radiotherapy equipment, more lines of systemic therapy, more clinic visits, palliative care services, and psychological support. Furthermore, the oncological services and facilities in LMICs are already limited; during the pandemic, those available may have been diverted to isolation centers or closed down altogether. Therefore, delay in treatment may result in a much poorer prognosis in LMICs compared to high-income countries [20].

Some other suggested practices for the successful delivery of oncological care without exposing patients to unnecessary harm were good communication, efficient infection control measures, displaying explicit instructions, and making available sanitizing liquids for all healthcare personnel and patients. Even when outside of cancer care facilities, patients should be advised to wear a mask, wash hands frequently, and practice social distancing. Telemedicine was expected to decrease patient exposure to hospital settings. Visiting patients needed to be screened and triaged carefully and all infected patients needed to be promptly shifted to isolation facilities to avoid exposure of other patients and healthcare workers.

Even when the healthcare system is functioning without the stress of a pandemic, the financial burden of cancer treatments is a recognized issue globally. LMICs can tackle this issue by replacing costly chemotherapeutic agents with biosimilars that have comparable efficacy. Currently, there are six approved trastuzumab biosimilars, which have shown equivalent efficacy to trastuzumab [21]. The wide distribution and utilization of such biosimilars in LMIC are encouraged to reduce financial burden [22].

\section{Low and Middle-income Countries: Morocco, Gaza, Ghana, India, The Philippines, Egypt, and Africa}

Many LMICs have published their challenges, experiences, and general recommendations with regards to general oncological care. Apart from a limited number of cancer care facilities, most cancer care was available in urban settings. Countrywide lock-downs, travel bans, and restricted hours of operation resulted in limited access to oncologic care. Under usual circumstances, cancer patients in Gaza require Israeli travel permits to leave Gaza and access treatment. Following travel restrictions, the mobility of these patients was greatly reduced and thus they avoided leaving Gaza for fear of not being able to return home, while some were forced to stay away from home to continue treatment. Patients were concerned about catching COVID-19 in the hospital setting, as overcrowding of waiting areas did not allow access to clean toilets and social distancing requirements could not be met [6]. Already burdened cancer care facilities were used as makeshift COVID-19 testing facilities and some were shut down due to resource allocation, greatly impacting the continuity of care. COVID-19 was also stigmatized and patients had started to fear COVID-19 more than cancer itself. The stigmatization prevented the patients from revealing their travel and exposure history which compromised the safety of the healthcare staff [23].

Though infection rates appeared to be lower in severely resource-constrained environments such as Africa [7], the speed with which the infected were identified and isolated was also low, thus putting the healthcare providers at risk. Though patients and providers were aware of the need for PPE, the lack of availability of PPE, medical consumables, and life support equipment, as well as the absence of health insurance to cover expenses, all led to great difficulty for both patients and the healthcare staff. Due to these limitations, a good number of healthcare workers got infected, were restricted to quarantine or isolation, or even died, further complicating the delivery of care [24].

Furthermore, limited external funding for an indefinite period inhibited research and resulted in a paucity of outcomes data in cancer patients during the pandemic, especially in LMICs [25]. This means that research on COVID-19 and its effect on cancer or vice versa will be limited from LMICs and greatly compromise our capacities to guide care in the future.

Breast cancer management in most countries was dictated by government mandates, institutional guidelines, or adaptation of guidelines from international organizations. No unified standard management plan could serve the global needs. Recommendations were made to guide care in all three fields 
of management including surgical, medical, and radiation oncology. It is recommended that in the case of COVID-19 and cancer, the infection should be prioritized and all treatment be halted unless the patient attains an asymptomatic state. It was unanimously suggested to delay or cancel elective procedures with the approval of the patient and the multi-disciplinary teams [17, 22, 23, 26-31].

For medical oncology, IV regimens were shifted to oral. In certain places, oncology teams were split into two: one to deal with infected patients with cancer and the other to deal with uninfected cancer patients. This plan helped with the distribution of workload, judicious use of PPE, and offered greater safety to the non-infected cancer patients [30]. Adjuvant radiotherapy regimens were also altered based on the cancer type and stage. Radiation therapy was sometimes shortened, made less intense, and/or shifted to alternate protocols that did not require in-patient admission. Low-risk patients and those where benefit from radiation was limited were encouraged to omit the modality. Data from the Egyptian literature recommended delaying all adjuvant radiotherapy except in high-risk breast cancer patients, including those with T3-4 tumors, clinically N2-3 patients, those who had triple negative disease, or those who were diagnosed young age. In general, the recommended radiation dose for adjuvant treatment was $40 \mathrm{~Gy} / 15$ fractions, with a possible concomitant boost of $200 \mathrm{cGy} / 5-8$ fractions to the primary tumor site in patients who had breast conserving surgery [31]. Routine clinic follow-ups and postoperative clinic patients were shifted to telehealth wherever possible.

To the best of our knowledge, Morocco was the only LMIC that published a standardized protocol for the treatment of breast cancer patients during the COVID-19 pandemic. For patients currently under treatment, their guidelines were similar to those of major leading organizations. They specifically recommended spacing out treatment protocols, replacing cisplatin-based therapies with carboplatin or oxaliplatin-based regimens wherever possible, advocated for the use of q3weekly chemotherapy regimens and the use of granulocyte-colony-stimulating factors (GCSF) to avoid neutropenia. They suggested postponing regular screening for breast cancer and deferment of surgery for carcinoma in situ and low-grade tumors. It was suggested that adjuvant radiation therapy be delayed for low-risk disease and hypofractionated RT be preferred for patients in whom radiation cannot be postponed. They further proposed the use of oral therapies for metastatic breast cancer patients [27] and replacing physical consultations with teleconsultations. For patients receiving palliative care, they recommended planning therapeutic spacing/deescalation whenever possible. Breast cancer patients who were being seen for surveillance alone were advised to present to the clinic in person only in case of development of symptoms of recurrence.
For all new cases, a risk-benefit assessment was advised based on urgency and need. For hospitalized patients, steps to prevent exposure and minimize expense were suggested including advising patients to bring their own alcohol-based hand sanitizers and blankets, decreasing family visits, limiting the number of doctors visiting the bedside, and provision of isolation facilities for patients who develop symptoms of COVID-19 infection [17, 22, 23, 26-31].

\section{Our Institutional Response-the Aga Khan University Hospital, Karachi, Pakistan}

Despite being the regional neighbor to China, Pakistan faced the peak of COVID-19 cases much later which allowed the country to take early measures in tackling the disease. Pakistan reported its first case of COVID-19 on February 26,2020 . In the early days, some of the provinces implemented a complete lockdown while others enforced a partial "smart" lockdown. Smart lockdown refers to a limited closure of non-essential facilities of the city while allowing the essential business and services to continue operating with predecided hours and days. The government prepared a comprehensive list of essential businesses that were allowed to operate including banks, hospitals, grocery stores, etc. while luxury/leisure businesses and services such as cinemas, restaurants, salons, and shopping malls were shut down. Furthermore, the timings and days of operation were also reduced to limit exposure. Isolation centers were set up both within the hospitals and at remote places and extensive screening, testing, and triage systems were set up.

At our institution, the Aga Khan University hospital, comprehensive, multidisciplinary guidelines were developed by modifying the existing COVID resources from the Society of Surgical Oncology (SSO) and American Society of Breast Surgeons (ASBrS) to suit the needs of our resource-limited healthcare structure. These guidelines were in line with the ethical framework provided by the Center of Biomedical Ethics and Culture (CBEC) at Sindh Institute of Urology and Transplantation (SIUT), Pakistan. These guidelines were approved by all stakeholders and were circulated to all members of the oncological care team including surgical, medical, and radiation oncology.

After careful review of each case, patients were triaged into three priority categories. Priority A patients were those where oncologic care could not be delayed such as those with malignant phyllodes tumor, angiosarcoma, or a post-operative complication that warranted a second emergent operation. Those patients where a delay in intervention by $4-8$ weeks was not expected to adversely affect the outcome were categorized as priority B. These included patients who could be started on neoadjuvant therapy, such that surgery could be delayed somewhat. Patients for whom treatment could be 
safely delayed till after the pandemic were labeled as priority $\mathrm{C}$ and included those with hormone positive tumors, who were responsive to neoadjuvant endocrine therapy [32]. Treatment course of all patients was decided based on their priority category as determined by the weekly virtual multidisciplinary tumor board.

All patients were screened at entry points to our facility where a risk assessment was done; wearing a mask was required. Telephonic, WhatsApp, and Zoom communication options were added to traditional in-person clinics to reduce patient need to leave home and limit exposure to COVID-19 infection. Follow-up patients had virtual consultations and all routine and screening checkups were deferred initially by 3 months. Follow-up patients with unresolved issues were invited to visit the onsite clinic. Steps were taken to protect these patients by spacing out appointment times to ensure no crowding in waiting areas, modifying the waiting area seating arrangement to maintain social distancing, and providing hand sanitizer dispensers at convenient locations.

All healthcare workers in oncology were divided into three teams which worked on a rotational schedule. Two teams worked regularly, and the third team was kept as a backup in case one team had to quarantine themselves. Schedules were drafted in a way that every worker got at least 1 to 2 days off during the week.

Initially, all elective surgeries including cancer surgery were put on hold for about 2 weeks due to escalating cases of COVID-19 in the country that also affected our employees. Within 1-2 weeks, a clear plan for the OR was devised to preserve personnel and supplies. A gradual phased reopening plan of the OR was carefully planned by all stakeholders. The operating schedule was modified to keep in line with the institutional/infection control guidelines. Each case had to be approved by the Section Heads, Chairs of the Department of Surgery and Anesthesia before an operating room could be allocated. An OR suite consisting of three negative pressure operating rooms was quickly designed and developed for COVID-positive or COVID-unknown emergency patients. Each patient undergoing elective surgery underwent a mandatory COVID-19 test and if infected, surgical care was deferred based on institutional/infection control guidelines.

With a decline in COVID-19 cases, we are now in the process of transitioning back into our normal operating schedule. From no elective surgeries in March 2020, we now have elective cases from 8 am-5 pm on weekdays; on Saturdays, the OR is still not open for elective cases. This has happened gradually in 8 phases. The COVID ORs are now in the process of being turned back into regular operating rooms and only one OR with a dedicated recovery area will remain as a negative pressure COVID operating suite. We are cognizant of the fact that Pakistan overall has done well so far; however, with cooler temperatures expected and schools reopening, a second surge is expected. We are aware of the fact that patients who were deferred earlier must receive care during this window of opportunity before the second surge arrives and we are therefore facilitating care as necessary.

We were able to manage our patient load efficiently because we quickly adapted to the evolving situation and our management protocol was implemented promptly. In addition, we had clear, daily communication between the COVID response center, various providers as well as the leadership of the hospital/university, clinicians, house-staff, and nursing.

\section{Conclusion}

The COVID-19 pandemic has challenged the global healthcare community like never before. The global community had to quickly adapt to the evolving situation and guidelines were put forward, mostly by the developed world. Some LMICs were quick to adapt these guidelines for their use. Based on Western data, there appear to be worse outcomes related to deviations from the standard of care; however, outcomes data from LMICs are lacking. Consideration of funding such outcomes research by funding agencies may facilitate preparation for future challenges.

\section{Declarations}

Conflict of Interest The authors declare no competing interests.

Human and Animal Rights and Informed Consent This article does not contain any studies with human or animal subjects performed by any of the authors.

\section{References}

1. Ologunde R, Maruthappu M, Shanmugarajah K, Shalhoub J. Surgical care in low and middle-income countries: burden and barriers. Int J Surg Lond Engl. 2014;12(8):858-63.

2. Dube PN, Sakle NS, Dhawale SA, More SA, Mokale SN. Synthesis, biological investigation and docking study of novel chromen derivatives as anti-cancer agents. Anticancer Agents Med Chem. 2019;19(9):1150-60.

3. Tagliamento M, Lambertini M, Genova C, Barisione E, De Maria A, Grosso M, et al. Call for ensuring cancer care continuity during COVID-19 pandemic. ESMO Open. 2020;5(3).

4. Zhang L, Zhu F, Xie L, Wang C, Wang J, Chen R, et al. Clinical characteristics of COVID-19-infected cancer patients: a retrospective case study in three hospitals within Wuhan, China. Ann Oncol Off J Eur Soc Med Oncol. 2020;31(7):894-901.

5. Verhagen LM, de Groot R, Lawrence CA, Taljaard J, Cotton MF, Rabie H. COVID-19 response in low- and middle-income countries: don't overlook the role of mobile phone communication. Int J Infect Dis IJID Off Publ Int Soc Infect Dis. 2020;99:334-7. 
6. AlWaheidi S, Sullivan R, Davies EA. Additional challenges faced by cancer patients in Gaza due to COVID-19. Ecancermedicalscience. 2020;14:ed100.

7. WHO coronavirus disease (COVID-19) dashboard. [cited 2020 Sep 27]. Available from: https://covid19.who.int

8. Estimating mortality from COVID-19. [cited 2020 Sep 25]. Available from: https:/www.who.int/news-room/commentaries/ detail/estimating-mortality-from-covid-19

9. Fauci AS, Lane HC, Redfield RR. Covid-19 - navigating the uncharted. N Engl J Med. 2020;382(13):1268-9.

10. SARS and MERS: recent insights into emerging coronaviruses | Nature Reviews Microbiology. [cited 2020 Sep 25]. Available from: https://www.nature.com/articles/nrmicro.2016.81.

11. Rajgor DD, Lee MH, Archuleta S, Bagdasarian N, Quek SC. The many estimates of the COVID-19 case fatality rate. Lancet Infect Dis. 2020;20(7):776-7.

12. EpiCentro. Characteristics of COVID-19 patients dying in Italy. [cited 2020 Sep 25]. Available from: https://www.epicentro.iss.it/ en/coronavirus/sars-cov-2-analysis-of-deaths

13. Rothan HA, Byrareddy SN. The epidemiology and pathogenesis of coronavirus disease (COVID-19) outbreak. J Autoimmun. 2020;109:102433.

14. Kuderer NM, Choueiri TK, Shah DP, Shyr Y, Rubinstein SM, Rivera DR, et al. Clinical impact of COVID-19 on patients with cancer (CCC19): a cohort study. Lancet Lond Engl. 2020;395(10241):1907-18.

15. Pino L, Perez C, Cardona A, Triana I. Cancer center recommendations to mitigate COVID-19 impact in patients with cancer: lowresource settings version. JCO Glob Oncol. 2020;6:569-70.

16. Braunstein LZ, Gillespie EF, Hong L, Xu A, Bakhoum SF, Cuaron $\mathrm{J}$, et al. Breast radiation therapy under COVID-19 pandemic resource constraints - approaches to defer or shorten treatment from a comprehensive cancer center in the United States. Adv Radiat Oncol. 2020;5(4):582-8.

17. Belkacemi Y, Grellier N, Ghith S, Debbi K, Coraggio G, Bounedjar $\mathrm{A}$, et al. A review of the international early recommendations for departments organization and cancer management priorities during the global COVID-19 pandemic: applicability in low- and middleincome countries. Eur J Cancer Oxf Engl 1990. 2020;135:130-46.

18. Zhan Q-H, Fu J-Q, Fu F-M, Zhang J, Wang C. Survival and time to initiation of adjuvant chemotherapy among breast cancer patients: a systematic review and meta-analysis. Oncotarget. 2018;9(2):2739-51.

19. Tfayli A, Temraz S, Abou Mrad R, Shamseddine A. Breast cancer in low- and middle-income countries: an emerging and challenging epidemic. J Oncol. 2010;2010:490631.

20. Anderson BO, Yip C-H, Smith RA, Shyyan R, Sener SF, Eniu A, et al. Guideline implementation for breast healthcare in low-income and middle-income countries: overview of the Breast Health Global Initiative Global Summit 2007. Cancer. 2008;113(8 Suppl):2221-43.

21. Miller EM, Schwartzberg LS. Biosimilars for breast cancer: a review of HER2-targeted antibodies in the United States. Ther Adv Med Oncol. 2019;11:1758835919887044.

22. Elghazawy H, Bakkach J, Zaghloul MS, Abusanad A, Hussein MM, Alorabi M, et al. Implementation of breast cancer continuum of care in low- and middle-income countries during the COVID-19 pandemic. Future Oncol. 2020;fon-2020-0574.

23. Lying COVID-19 patients caused deaths among doctors, DOH says. CNN Philippines. Available from: https://cnnphilippines.com/news/ 2020/3/27/COVID-19-lying-patients-doctor-deaths.html

24. Restivo A, De Luca R, Spolverato G, Delrio P, Lorenzon L, D’Ugo $\mathrm{D}$, et al. The need of COVID19 free hospitals to maintain cancer care. Eur J Surg Oncol. 2020 Jun 1;46(6):1186-7.

25. Cancer today. [cited 2020 Sep 27]. Available from: http://gco.iarc. fr/today/home

26. Kugbey N, Ohene-Oti N, Vanderpuye V. COVID-19 and its ramifications for cancer patients in low-resource settings: Ghana as a case study. Ecancermedicalscience. 2020;14:ed99.

27. Ismaili N, El Majjaoui S. Management of breast cancer during COVID-19 pandemic in Morocco. Breast J. 2020;26(8):1618-9.

28. Mrabti H, Berrada N, Raiss G, Ettahri H, Abahssain H, Bourhafour $\mathrm{M}$, et al. Cancer management challenge in a developing country in COVID-19 pandemic: reflection of a group of Moroccan oncologists. Future Oncol Lond Engl. 2020;16(26):2007-16.

29. The tale of two C's, cancer and COVID-19: oncologist's viewpoint from India $\mid$ Asian Pacific Journal of Cancer Care. [cited 2020 Sep 27]. Available from: http://www.waocp.com/journal/index.php/ apjcc/article/view/411

30. Okeke M, Oderinde O, Liu L, Kabula D. Oncology and COVID19: perspectives on cancer patients and oncologists in Africa. Ethics Med Public Health. 2020;14:100550.

31. Elkhouly EA, Salem RH, Haggag M. Should cancer treatment be continued during the COVID-19 pandemic? A single Egyptian institution experience. Ecancermedicalscience. 2020;14:1077.

32. Sattar AK, Shahzad H, Jabbar AA, et al. A multidisciplinary approach to triage patients with breast disease during the COVID-19 pandemic: experience from a tertiary care center in the developing world. Cancer Rep. 2020;e1309. https://doi.org/10.1002/cnr2.1309 (accepted for publication)

Publisher's Note Springer Nature remains neutral with regard to jurisdictional claims in published maps and institutional affiliations. 